Erratum

\title{
Role of Sirolimus in renal tubular apoptosis in response to unilateral ureteral obstruction: Erratum
}

\author{
Mei Yang1, Yang-yang Zhuang1, Wei-wei Wang1, Hai-ping Zhu1, Yan-jie Zhang1, Sao-ling Zheng2, \\ Yi-Rrong Yang ${ }^{2}$, Bi-Cheng Chen ${ }^{3}$, Peng $\mathrm{Xia}^{2}{ }^{\circledR}$, Yan Zhang ${ }^{2}$ \\ 1. Department of Intensive Care Unit, the First Affiliated Hospital of Wenzhou Medical University, Wenzhou, Zhejiang Province, China 325015 \\ 2. Transplantation centre, the First Affiliated Hospital of Wenzhou Medical University, Wenzhou, Zhejiang Province, China 325015 \\ 3. Zhejiang Provincial Top Key Discipline in Surgery, Wenzhou Key Laboratory of Surgery, Department of Surgery, The First Affiliated Hospital of Wenzhou Medical \\ University, Wenzhou, Zhejiang Province 325015, China
}

$\square$ Corresponding authors: Peng Xia, MD, Transplantation centre, the First Affiliated Hospital of Wenzhou Medical University, Wenzhou, Zhejiang, China 325015. Phone: +86-13857753169, E-mail: pengxia602@163.com and Yan Zhang, MD, PHD, Transplantation centre, the First Affiliated Hospital of Wenzhou Medical University, Wenzhou, Zhejiang, China 325015. Phone: +86-15858583023, E-mail: biobabry@163.com

(C) The author(s). This is an open access article distributed under the terms of the Creative Commons Attribution License (https://creativecommons.org/licenses/by/4.0/). See http:/ /ivyspring.com/terms for full terms and conditions.

Published: 2022.03 .03

Corrected article: Int J Med Sci 2018; 15(13): 1433-1442. doi: 10.7150/ijms.26954.

In our paper [1], Figure 1 and Figure 2 should be corrected as follows.
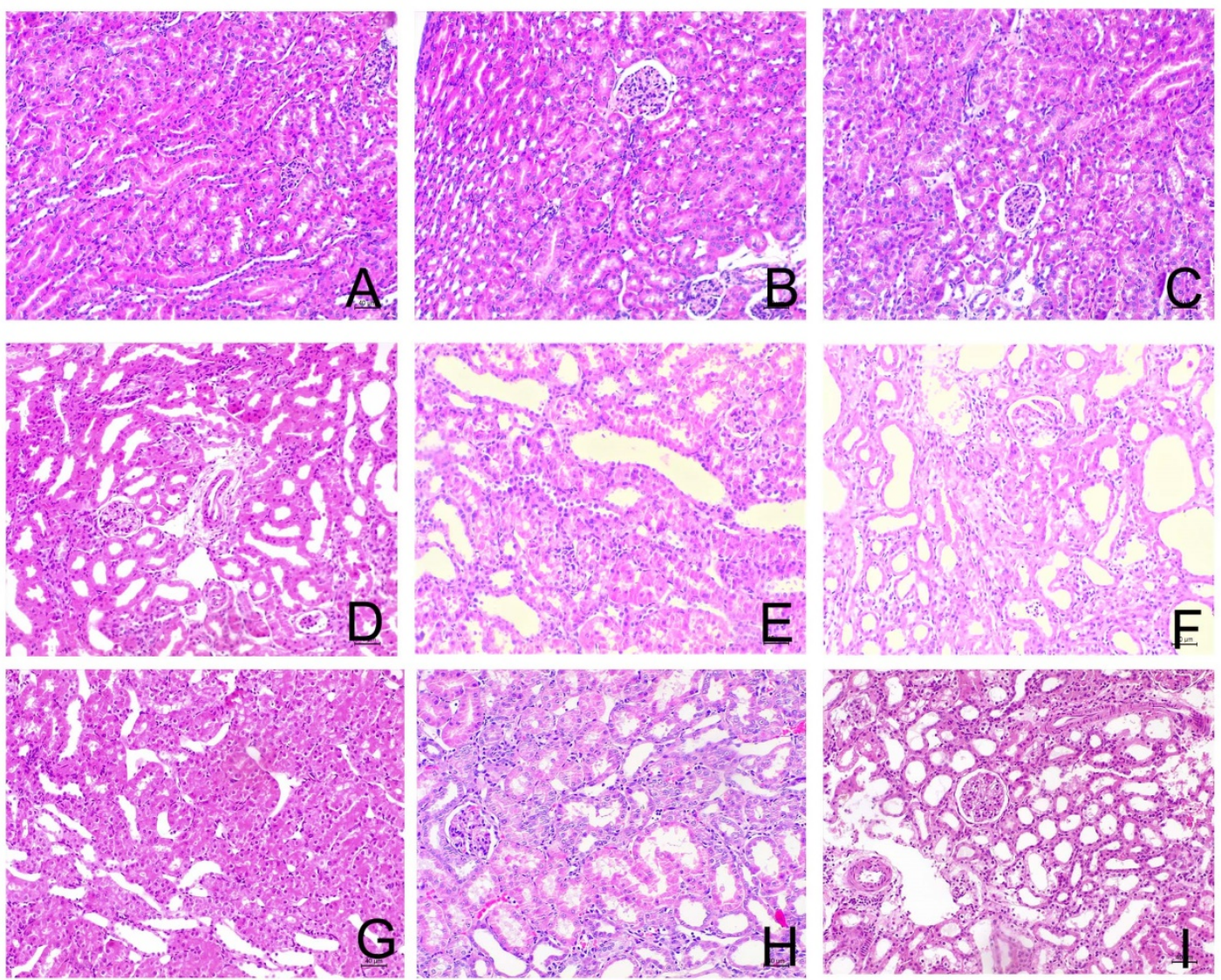

Figure 1. Sirolimus attenuated the histological changes in the obstructed kidney induced by UUO. Representative hematoxylin-eosin staining micrographs of (A, B, C) Sham group, (D, E, F) UUO group and (G, H, I) Sirolimus group. Original magnification $\times 200$. 

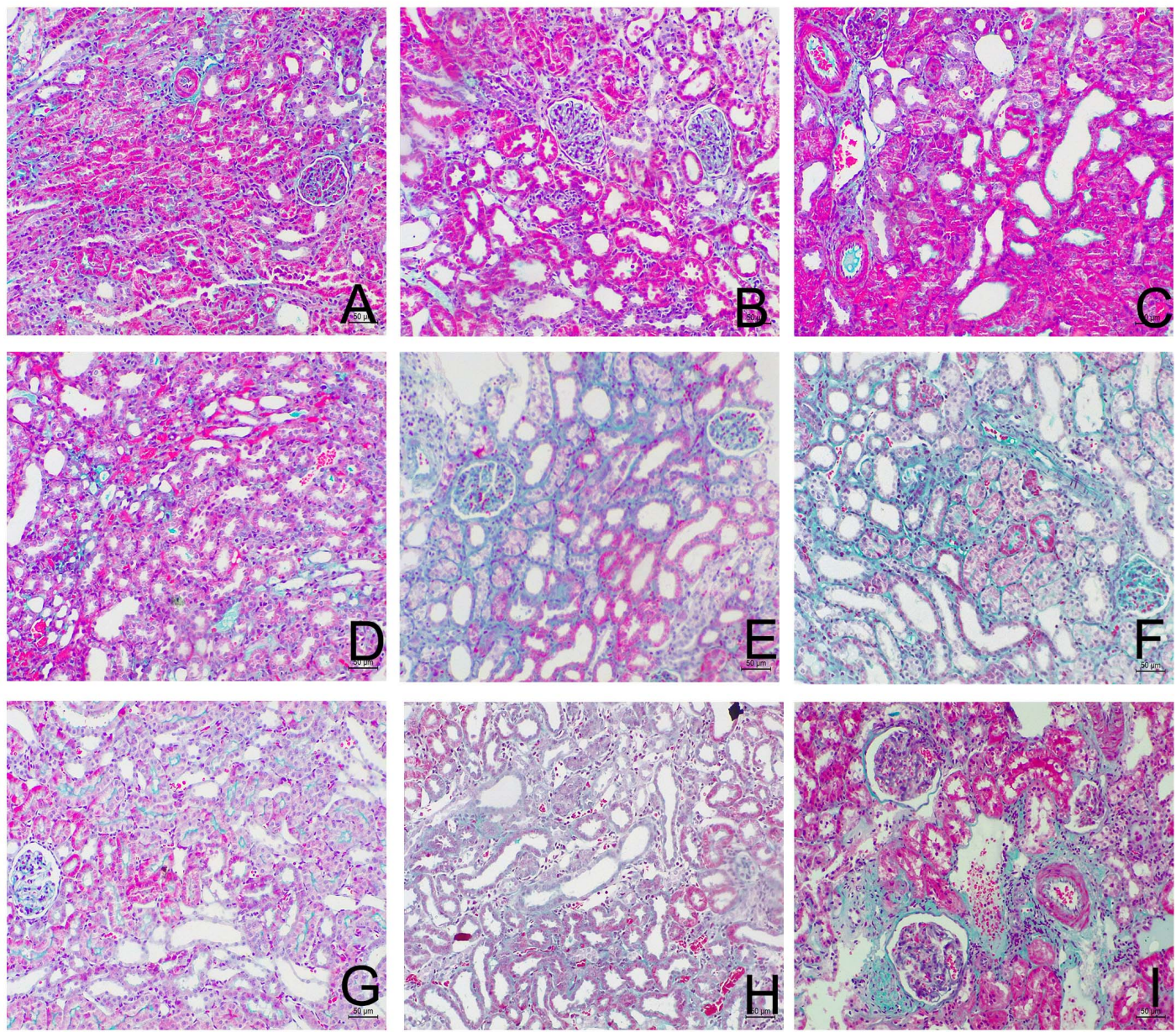

Figure 2. Sirolimus attenuated the interstitial collagen deposition in the obstructed kidney induced by UUO. Representative Masson's trichrome staining micrographs of (A, B, C) Sham group, (D, E, F) UUO group and (G, H, I) Sirolimus group. Original magnification $\times 200$.

\section{References}

[1] Yang M, Zhuang Yy, Wang Ww, Zhu Hp, Zhang Yj, Zheng Sl, Yang YR, Chen BC, Xia P, Zhang Y. Role of Sirolimus in renal tubular apoptosis in response to unilateral ureteral obstruction. Int J Med Sci 2018; 15(13):1433-1442. doi:10.7150/ijms.26954. 